Pacific

Journal of

Mathematics

\author{
WEIL REPRESENTATIONS OF \\ FINITE GENERAL LINEAR GROUPS \\ AND FINITE SPECIAL LINEAR GROUPS
}

Pham HuU TieP 


\title{
WEIL REPRESENTATIONS OF FINITE GENERAL LINEAR GROUPS AND FINITE SPECIAL LINEAR GROUPS
}

\author{
PHAM HUU TIEP
}

Dedicated to the memory of Professor R. Steinberg

Let $q$ be a prime power and let $G_{n}$ be the general linear group $\mathrm{GL}_{n}(q)$ or the special linear group $\operatorname{SL}_{n}(q)$, with $n \geq 4$. We prove two characterization theorems for the Weil representations of $G_{n}$ in any characteristic coprime to $q$, one in terms of the restriction to a standard subgroup $G_{n-1}$, and another in terms of the restriction to a maximal parabolic subgroup of $G_{n}$.

\section{Introduction}

The so-called Weil representations were introduced by A. Weil [1964] for classical groups over local fields. Weil mentioned that the finite field case may be considered analogously. This was developed in detail by R. E. Howe [1973] and P. Gérardin [1977] for characteristic zero representations. The same representations, still in characteristic zero, were introduced independently by I. M. Isaacs [1973] and H. N. Ward [1972] for symplectic groups $\operatorname{Sp}_{2 n}(q)$ with $q$ odd, and by G. M. Seitz [1975] for unitary groups. (These representations for $\operatorname{Sp}_{2 n}(p)$ were also constructed in [Bolt et al. 1961].) Weil representations of finite symplectic groups $\operatorname{Sp}_{2 n}(q)$ with $2 \mid q$ were constructed by R. M. Guralnick and the author in [Guralnick and Tiep 2004]. Weil representations attract much attention because of their many interesting features; see, for instance, [Dummigan 1996; Dummigan and Tiep 1999; Gow 1989; Gross 1990; Scharlau and Tiep 1997; 1999; Tiep 1997a; 1997b, Zalesski 1988].

The construction of the Weil representations may be found in [Howe 1973; Gérardin 1977; Guralnick and Tiep 2004; Seitz 1975], etc. In particular, in the case of general and special linear groups, they can be constructed as follows. Let $W=\mathbb{F}_{q}^{n}$ with $n \geq 3$, and let $\tilde{\zeta} \in \mathbb{C}$, respectively $\zeta \in \mathbb{F}_{q}$, be a fixed primitive $(q-1)$-th root of unity. Then $\operatorname{SL}(W)=\operatorname{SL}_{n}(q)$ has $q-1$ complex Weil representations, which are

The author gratefully acknowledges the support of the NSF (grant DMS-1201374) and the Simons Foundation Fellowship 305247.

MSC2010: 20C15, 20C20, 20C33.

Keywords: Weil representations, finite general linear groups, finite special linear groups. 
the nontrivial irreducible constituents of the permutation representation of $\operatorname{SL}(W)$ on $W \backslash\{0\}$. The characters of these representations are $\tau_{n, q}^{i}$, where $0 \leq i \leq q-2$ and

$$
\tau_{n, q}^{i}(g)=\frac{1}{q-1} \sum_{k=0}^{q-2} \tilde{\zeta}^{i k} q^{\operatorname{dim} \operatorname{Ker}\left(g-\zeta^{k} \cdot 1_{W}\right)}-2 \delta_{0, i} .
$$

Similarly, $\mathrm{GL}(W)=\mathrm{GL}_{n}(q)$ has $(q-1)^{2}$ complex Weil representations, which are the $q-1$ nontrivial irreducible constituents of the permutation representation of GL(W) on $W \backslash\{0\}$, tensored with one of the $q-1$ representations of degree 1 of $\operatorname{GL}_{n}(q)$. If we fix a character $\alpha$ of order $q-1$ of $\mathrm{GL}_{n}(q)$, then the characters of these representations are $\tau_{n, q}^{i, j}$, where $0 \leq i, j \leq q-2$ and

$$
\tau_{n, q}^{i, j}(g)=\left(\frac{1}{q-1} \sum_{k=0}^{q-2} \tilde{\zeta}^{i j} q^{\operatorname{dim} \operatorname{Ker}\left(g-\zeta^{k} \cdot 1_{W}\right)}-2 \delta_{0, i}\right) \cdot \alpha^{j}(g) .
$$

Note that $\tau_{n, q}^{i, j}$ restricts to $\tau_{n, q}^{i}$ over $\operatorname{SL}_{n}(q)$.

From now on, let us fix a prime $p$, a power $q$ of $p$, and an algebraically closed field $\mathbb{F}$ of characteristic $\ell$ not equal to $p$. If $G$ is a finite (general or special) linear group, unitary group, or symplectic group, then by a Weil representation of $G$ over $\mathbb{F}$, we mean any composition factor of degree $>1$ of a reduction modulo $\ell$ of a complex Weil representation of $G$. As it turns out, another important feature of the Weil representations is that, with very few small exceptions, Weil representations are precisely the irreducible $\mathbb{F} G$-representations of the first few smallest degrees (larger than 1); see [Brundan and Kleshchev 2000; Guralnick et al. 2002; 2006, Guralnick and Tiep 1999, 2004, Hiss and Malle 2001; Tiep and Zalesski 1996]. Aside from this characterization by degree, Weil representations can also be recognized by various conditions imposed on their restrictions to standard subgroups, or parabolic subgroups. This was done in the case where $G$ is a symplectic group or a unitary group, in [Tiep and Zalesski 1997] for complex representations and in [Guralnick et al. 2002, 2006, Guralnick and Tiep 2004] for modular representations (in cross characteristics). However, the case where $G$ is a general or special linear group has not been treated. Perhaps one of the reasons for the absence until now of such characterizations (as regards the restriction to a parabolic subgroup) is that the obvious analogue of [Guralnick et al. 2002, Corollary 12.4] fails in this case; see Example 4.1.

We now fix $W=\mathbb{F}_{q}^{n}$ with a basis $\left(e_{1}, \ldots, e_{n}\right)$, and consider $G_{n}=G=\operatorname{GL}(W)$ or $\mathrm{SL}(W)$. By a standard subgroup $G_{m}$ in $G_{n}$, where $1 \leq m \leq n-1$, we mean (any $G$-conjugate of) the subgroup

$$
G_{m}=\operatorname{Stab}_{G_{n}}\left(\left\langle e_{1}, \ldots, e_{m}\right\rangle_{\mathbb{F}_{q}}, e_{m+1}, \ldots, e_{n}\right) .
$$

Next, we fix a primitive $p$-th root of unity $\epsilon \in \mathbb{C}$. A maximal parabolic subgroup of $G$ is conjugate to

$$
P=\operatorname{Stab}_{G}\left(\left\langle e_{1}, \ldots, e_{k}\right\rangle_{\mathbb{F}_{q}}\right)
$$


for some $1 \leq k \leq n-1$. The unipotent radical $Q$ of $P$ is

$$
\left\{[X]:=\left(\begin{array}{cc}
I_{k} & X \\
0 & I_{n-k}
\end{array}\right) \mid X \in M_{k, n-k}\left(\mathbb{F}_{q}\right)\right\},
$$

where $M_{a, b}\left(\mathbb{F}_{q}\right)$ is the space of all $a \times b$ matrices over $\mathbb{F}_{q}$. Any irreducible Brauer character of $Q$ can then be written in the form

$$
\beta_{Y}:[X] \mapsto \epsilon^{\operatorname{Tr}_{\mathbb{F} q / \mathbb{F} p} \operatorname{tr}(X Y)}
$$

for a unique $Y \in M_{n-k, k}\left(\mathbb{F}_{q}\right)$. We define the rank of $\beta_{Y}$ to be the rank of the matrix $Y$.

The main results of the paper are the following theorems, which characterize the Weil representations of finite general and special linear groups in terms of their restrictions to a standard or maximal parabolic subgroup.

Theorem A. Let $q$ be a prime power, $n \geq 5$, and for any integer $m \geq 4$, let $G_{m}$ denote the general linear group $\mathrm{GL}_{m}(q)$ or the special linear group $\operatorname{SL}_{m}(q)$. Consider the standard embedding of $G_{m}$ in $G_{n}$ when $n>m$. Let $\mathbb{E}$ be an algebraically closed field of characteristic zero or characteristic coprime to $q$. Then for any finite-dimensional irreducible $\mathbb{F} G_{n}$-representation $\Phi$, the following statements are equivalent:

(i) Either $\operatorname{deg} \Phi=1$, or $\Phi$ is a Weil representation of $G_{n}$.

(ii) $\Phi$ has property $(\mathcal{W})$; that is, if $\Psi$ is any composition factor of the restriction $\left.\Phi\right|_{G_{n-1}}$, then either $\operatorname{deg} \Psi=1$, or $\Psi$ is a Weil representation of $G_{n-1}$.

(iii) For some $m$ with $4 \leq m \leq n-1$, every composition factor of the restriction $\left.\Phi\right|_{G_{m}}$ either is a Weil representation or has degree 1.

(iv) For every $m$ with $4 \leq m \leq n-1$, every composition factor of the restriction $\left.\Phi\right|_{G_{m}}$ either is a Weil representation or has degree 1.

Theorem B. Let $q$ be a prime power, $n \geq 4$, and let $G$ denote the general linear group $\mathrm{GL}_{n}(q)$ or the special linear group $\mathrm{SL}_{n}(q)$. Let $\mathbb{F}$ be an algebraically closed field of characteristic zero or characteristic coprime to $q$. Let $W=\mathbb{F}_{q}^{n}$ denote the natural $G$-module, and let $P_{k}$ be the stabilizer of a $k$-dimensional subspace of $W$ in $G$, where $2 \leq k \leq n-2$. Then for any finite-dimensional irreducible $\mathbb{F} G_{n}$-representation $\Phi$, the following statements are equivalent:

(i) Either $\operatorname{deg} \Phi=1$, or $\Phi$ is a Weil representation of $G_{n}$.

(ii) $\Phi$ has property $\left(\mathcal{P}_{k}\right)$; that is, the restriction $\left.\Phi\right|_{Q}$ to the unipotent radical $Q$ of $P_{k}$ contains only irreducible $\mathbb{F} Q$-representations of rank $\leq 1$.

Note that the definitions of properties $(\mathcal{W})$ and $\left(\mathcal{P}_{k}\right)$ do not depend on the choice of the particular standard or parabolic subgroup. Our subsequent proofs also make use of another local property $(\mathcal{Z})$, which is defined by the condition (2-1) in Section 2. 
Theorem B has already been used by A. E. Zalesski [ $\geq 2015]$ in his recent work.

Throughout the paper, we will say that an ordinary or Brauer character $\varphi$ of $\mathrm{GL}_{n}(q)$ or $\mathrm{SL}_{n}(q)$ has property $(\mathcal{W})$, or $\left(\mathcal{P}_{k}\right)$, if a representation affording $\varphi$ does as well. The notation $\operatorname{IBr}(X)$ denotes the set of irreducible Brauer characters of a finite group $X$ in characteristic $\ell$. If $Y$ is a subgroup of a finite group $X$, and $\Phi$ is an $\mathbb{E} X$-representation and $\Psi$ is an $\mathbb{E} Y$-representation, then $\left.\Phi\right|_{Y}$ is the restriction of $\Phi$ to $Y$, and $\Psi^{Y}$ is the $\mathbb{F} X$-representation induced from $\Psi$, with similar notation for ordinary and Brauer characters, as well as for modules. If $\chi$ is a complex character of $X$, then $\chi^{\circ}$ denotes the restriction of $\chi$ to the set of $\ell^{\prime}$-elements in $X$.

\section{Local properties and Weil representations}

A key ingredient of our inductive approach is the following statement:

Proposition 2.1. Let $G=G_{n}=\mathrm{GL}_{n}(q)$ with $n \geq 5$ and let $\Phi$ be an $\mathbb{F} G$-representation. Let $K=G_{4}$ be a standard subgroup of $G$. Then the following statements hold.

(i) If $\Phi$ has property $\left(\mathcal{P}_{k}\right)$ for some $2 \leq k \leq n-2$, then the $\mathbb{F} K$-representation $\left.\Phi\right|_{K}$ has property $\left(\mathcal{P}_{2}\right)$.

(ii) If $\left.\Phi\right|_{K}$ has property $\left(\mathcal{P}_{2}\right)$, then $\Phi$ has property $\left(\mathcal{P}_{k}\right)$ for all $2 \leq k \leq n-2$. Proof. Consider

$$
\begin{gathered}
P=\operatorname{Stab}_{G}\left(\left\langle e_{1}, \ldots, e_{k}\right\rangle_{\mathbb{F}_{q}}\right), \\
K=\operatorname{Stab}_{G}\left(\left\langle e_{1}, e_{2}, e_{k+1}, e_{k+2}\right\rangle_{\mathbb{F}_{q}}, e_{3}, \ldots, e_{k}, e_{k+3}, \ldots, e_{n}\right) .
\end{gathered}
$$

Then $P_{2}:=P \cap K$ plays the role of the second parabolic subgroup of $K$, the stabilizer in $K$ of the plane $\left\langle e_{1}, e_{2}\right\rangle_{\mathbb{F}_{q}}$, with unipotent radical $Q_{2}:=Q \cap K$. Let $\varphi$ denote the Brauer character of $\Phi$.

(i) Suppose that $\Phi$ has property $\left(\mathcal{P}_{k}\right)$. Consider any irreducible constituent $\beta=\beta_{Y}$ of $\left.\varphi\right|_{Q}$. By the assumption, $\operatorname{rank}(Y) \leq 1$. Writing

$$
Y=\left(\begin{array}{ll}
Y_{1} & Y_{2} \\
Y_{3} & Y_{4}
\end{array}\right)
$$

where $Y_{1}$ is $2 \times 2$, it is easy to see that the restriction $\left.\beta\right|_{Q_{2}}$ is just the character $\beta_{Y_{1}}$ of $Q_{2}$. Certainly, $\operatorname{rank}\left(Y_{1}\right) \leq \operatorname{rank}(Y)$. It follows that $\left.\Phi\right|_{K}$ has property $\left(\mathcal{P}_{2}\right)$.

(ii) Suppose that $\Phi$ does not possess property $\left(\mathcal{P}_{k}\right)$ for some $2 \leq k \leq n-2$. Then we can find an irreducible constituent $\beta=\beta_{Y}$ of $\left.\varphi\right|_{Q}$, where $\operatorname{rank}(Y)=: r \geq 2$. Note that the conjugation by the element $\operatorname{diag}(A, B)$ in the Levi subgroup

$$
L=\mathrm{GL}_{k}(q) \times \mathrm{GL}_{n-k}(q)
$$

of $P$ sends $\beta_{Y}$ to $\beta_{B^{-1} Y A}$. Replacing $\beta$ by a suitable $L$-conjugate, we may assume that the principal $r \times r$ submatrix of $Y$ is the identity matrix $I_{r}$. Now, in the notation 
of (i), we have $\left.\beta\right|_{Q_{2}}=\beta_{Y_{1}}$, where $Y_{1}=I_{2}$. But this violates property $\left(\mathcal{P}_{2}\right)$ for $\left.\Phi\right|_{K}$.

Corollary 2.2. Let $G=G_{n}=\mathrm{GL}_{n}(q)$ with $n \geq 5$ and let $\Phi$ be an $\mathbb{F} G$-representation. Let $H=G_{m}$ be a standard subgroup of $G$ for some $4 \leq m \leq n-1$. Then the following statements hold.

(i) If $\Phi$ has property $\left(\mathcal{P}_{k}\right)$ for some $2 \leq k \leq n-2$, then the $\mathbb{F} H$-representation $\left.\Phi\right|_{H}$ has property $\left(\mathcal{P}_{j}\right)$ for all $2 \leq j \leq m-2$.

(ii) If $\Phi_{H}$ has property $\left(\mathcal{P}_{j}\right)$ for some $2 \leq j \leq m-2$, then $\Phi$ has property $\left(\mathcal{P}_{k}\right)$ for all $2 \leq k \leq n-2$.

Proof. Consider a standard subgroup $K=G_{4}$ of $H$.

(i) By applying Proposition 2.1(i) to $\Phi,\left.\Phi\right|_{K}$ has property $\left(\mathcal{P}_{2}\right)$. Hence, by applying Proposition 2.1(ii) to $\left.\Phi\right|_{H},\left.\Phi\right|_{H}$ has property $\left(\mathcal{P}_{j}\right.$ ) for all $2 \leq j \leq m-2$.

(ii) By applying Proposition 2.1(i) to $\left.\Phi\right|_{H},\left.\Phi\right|_{K}$ has property $\left(\mathcal{P}_{2}\right)$. Hence, by applying Proposition 2.1(ii) to $\Phi, \Phi$ has property $\left(\mathcal{P}_{k}\right)$ for all $2 \leq k \leq n-2$.

We will also fix the following elements in $\operatorname{GL}_{n}(q)$ :

$$
\boldsymbol{x}=\left(\begin{array}{ll}
1 & 1 \\
0 & 1
\end{array}\right) \oplus I_{n-2}, \quad \boldsymbol{y}=\left(\begin{array}{ll}
1 & 1 \\
0 & 1
\end{array}\right) \oplus\left(\begin{array}{ll}
1 & 1 \\
0 & 1
\end{array}\right) \oplus I_{n-4}, \quad z=\left(\begin{array}{lll}
1 & 1 & 0 \\
0 & 1 & 1 \\
0 & 0 & 1
\end{array}\right) \oplus I_{n-3}
$$

If $\varphi$ is a Brauer character of $G$, we define

$$
\varphi[1]:=\varphi(1)-(q+1) \varphi(\boldsymbol{x})+q \varphi(\boldsymbol{y}), \quad \varphi[2]:=\varphi(\boldsymbol{y})-\varphi(z) .
$$

We will furthermore say that $\varphi$ (or any representation affording it) has property $(\mathcal{Z})$ if

$$
\varphi[1]=\varphi[2]=0 .
$$

Corollary 2.3. Let $G=\mathrm{GL}_{n}(q)$ or $\mathrm{SL}_{n}(q)$ with $n \geq 4$ and let $\Phi$ be a Weil representation of $G$ over $\mathbb{F}$. Then $\Phi$ has property $\left(\mathcal{P}_{k}\right)$ for all $2 \leq k \leq n-2$. If $n \geq 5$, then $\Phi$ has property $(\mathcal{W})$.

Proof. It suffices to prove the statement in the case where $\mathbb{F}=\mathbb{C}$ and furthermore $G=\mathrm{GL}_{n}(q)$, as $\tau_{n, q}^{i, j}$ restricts to $\tau_{n, q}^{i}$ over $\operatorname{SL}_{n}(q)$.

(i) First we consider the case $G=G L_{4}(q)$ and consider the parabolic subgroup $P=\operatorname{Stab}_{G}\left(\left\langle e_{1}, e_{2}\right\rangle_{\mathbb{F}_{q}}\right)$, with unipotent radical $Q$. It is easy to check that $\operatorname{IBr}(Q)$ consists of three $P$-orbits: $\left\{1_{Q}\right\}, \mathcal{O}_{1}$ of characters of rank 1 , and $\mathcal{O}_{2}$ of characters of rank 2; moreover,

$$
\left|\mathcal{O}_{1}\right|=\left(q^{2}-1\right)(q+1), \quad\left|\mathcal{O}_{2}\right|=\left(q^{2}-1\right)\left(q^{2}-q\right) .
$$

If $q \geq 3$, then $\left|\mathcal{O}_{2}\right|>\tau_{4, q}^{i, j}(1)$ for all $i, j$, whence $\left.\left(\tau_{4, q}^{i, j}\right)\right|_{Q}$ can afford only characters of rank $\leq 1$, and so we are done. 
Suppose that $q=2$, in which case there is only one complex Weil character $\chi=\tau_{4,2}^{1,1}$ of degree 14. We also consider the irreducible complex character $\rho$ of $G=\mathrm{GL}_{4}(2) \cong \mathrm{A}_{8}$ of degree 7. As $\left|\mathcal{O}_{1}\right|=9,\left|\mathcal{O}_{2}\right|=6$, and $Q \not \leq \operatorname{Ker}(\rho)$, we must have that

$$
\left.\rho\right|_{Q}=1_{Q}+\sum_{\lambda \in \mathcal{O}_{2}} \lambda
$$

For the aforementioned involutions $\boldsymbol{x}, \boldsymbol{y}$, we have that

$$
\chi(x)=6, \quad \chi(y)=2,
$$

whence $\boldsymbol{x}$ belongs to class $2 A$ and $\boldsymbol{y}$ belongs to class $2 B$ in the notation of [Conway et al. 1985]. It follows that

$$
\rho(\boldsymbol{x})=-1, \quad \rho(\boldsymbol{y})=3,
$$

and so

$$
\sum_{\lambda \in \mathcal{O}_{2}} \lambda(\boldsymbol{x})=-2, \quad \sum_{\lambda \in \mathcal{O}_{2}} \lambda(\boldsymbol{y})=2, \quad \sum_{\lambda \in \mathcal{O}_{1}} \lambda(\boldsymbol{x})=1, \quad \sum_{\lambda \in \mathcal{O}_{1}} \lambda(\boldsymbol{x})=-3 .
$$

Since

$$
\left.\chi\right|_{Q}=a \cdot 1_{Q}+b \sum_{\lambda \in \mathcal{O}_{1}} \lambda+c \sum_{\lambda \in \mathcal{O}_{2}} \lambda
$$

for some nonnegative integers $a, b, c$, we conclude that $(a, b, c)=(5,1,0)$, i.e., $\chi$ has property $\left(\mathcal{P}_{2}\right)$, as desired.

(ii) Now we consider the general case of $G=\operatorname{GL}_{n}(q)$ with $n \geq 5$, and consider a standard subgroup $H=\mathrm{GL}_{4}(q)$ and a standard subgroup $L=\mathrm{GL}_{n-1}(q)$ in $G$. Let $\tau_{n}$ denote the permutation character of $G$ on $\mathbb{F}_{q}^{n}$, so that

$$
\tau_{n}=\sum_{i=0}^{q-2} \tau_{n, q}^{i, 0}+2 \cdot 1_{G} .
$$

Note that $\left.\left(\tau_{n}\right)\right|_{L}=q \tau_{n-1}$, and so $\Phi$ has property $(\mathcal{W})$. Similarly, $\left.\left(\tau_{n}\right)\right|_{H}=q^{n-4} \tau_{4}$. Furthermore, according to (i), $\tau_{4}$ has property $\left(\mathcal{P}_{2}\right)$. It follows that $\left.\left(\tau_{n}\right)\right|_{H}$ also has property $\left(\mathcal{P}_{2}\right)$, and so, by Corollary $2.2(\mathrm{ii}), \tau_{n}$ has property $\left(\mathcal{P}_{k}\right)$. Consequently, $\tau_{n, q}^{i, 0}$ and $\tau_{n, q}^{i, j}$ also possess property $\left(\mathcal{P}_{k}\right)$.

Lemma 2.4. Let $G=\operatorname{GL}_{n}(q)$ or $\operatorname{SL}_{n}(q)$, where $n \geq 4$, and let $\Phi$ be a Weil representation of $G$ over $\mathbb{F}$. Then $\Phi$ has property $(\mathcal{Z})$.

Proof. Let $\varphi$ be the Brauer character of $\Phi$. It is well known, see [Guralnick and Tiep 1999] for instance, that $\varphi$ is a linear combination of the reduction modulo $\ell$ of some $\chi=\tau_{n, q}^{i, j}$ or $\tau_{n, q}^{i}$ (note that such reductions need not be irreducible) and a 
linear character of $G$. As any linear character has property $(\mathcal{Z})$, it suffices to show that $\chi$ has property $(\mathcal{Z})$. According to (1-2), we have

$\chi(1)=\frac{q^{n}-1}{q-1}-\delta_{0, i}, \quad \chi(\boldsymbol{x})=\frac{q^{n-1}-1}{q-1}-\delta_{0, i}, \quad \chi(\boldsymbol{y})=\chi(z)=\frac{q^{n-2}-1}{q-1}-\delta_{0, i}$,

which implies property $(\mathcal{Z})$ for $\chi$.

Proposition 2.5. Let $G=\mathrm{GL}_{n}(q)$ and $S=\mathrm{SL}_{n}(q) \leq G$ with $n \geq 4$. Let $\Phi$ be an $\mathbb{F} G$-representation and let $\Psi$ be an $\mathbb{F} S$-representation. Also, let $\mathcal{P}=\left(\mathcal{P}_{k}\right)$ for some $2 \leq k \leq n-2$, or $\mathcal{P}=(\mathcal{W})$.

(i) $\Phi$ has property $\mathcal{P}$ if and only if $\left.\Phi\right|_{S}$ has property $\mathcal{P}$.

(ii) $\Psi$ has property $\mathcal{P}$ if and only if $\Psi^{G}$ has property $\mathcal{P}$.

Proof. (a) First we consider the case $\mathcal{P}=\left(\mathcal{P}_{k}\right)$ and let $P$ be the stabilizer in $G$ of a $k$-space in the natural module $W=\mathbb{F}_{q}^{n}$, with unipotent radical $Q$. Note that $Q<P \cap S$. Furthermore, if $\mathcal{O}_{1}$ denotes the set of all Brauer irreducible characters of $Q$ of rank 1, then $\mathcal{O}_{1}$ forms a single $P$-orbit and also a single $P \cap S$-orbit.

It is clear that $\Phi$ has property $\left(\mathcal{P}_{k}\right)$ if and only if $\left.\Phi\right|_{S}$ has property $\left(\mathcal{P}_{k}\right)$, since $Q<S$. It is also clear that $\Psi$ has property $\left(\mathcal{P}_{k}\right)$ whenever $\Psi^{G}$ has property $\left(\mathcal{P}_{k}\right)$, since $\left.\Psi\right|_{Q}$ is a subquotient of $\left.\left(\Psi^{G}\right)\right|_{Q}$. Assume now that $\Psi$ has property $\left(\mathcal{P}_{k}\right)$ and affords the Brauer character $\psi$. Then, by the aforementioned discussion, $\left.\psi\right|_{Q}=a \cdot 1_{Q}+b \gamma$ for some integers $a, b \geq 0$ and $\gamma:=\sum_{\lambda \in \mathcal{O}_{1}} \lambda$. Note that we can find a cyclic subgroup $C \cong C_{q-1}$ of $P$ such that $G=S \rtimes C$, and again by the aforementioned discussion, $C$ preserves $\gamma$. As

$$
\left.\left(\psi^{G}\right)\right|_{Q}=\left.\sum_{c \in C}\left(\psi^{c}\right)\right|_{Q}
$$

we conclude that $\left.\left(\psi^{G}\right)\right|_{Q}=(q-1)\left(a \cdot 1_{Q}+b \gamma\right)$, and so $\Psi^{G}$ has property $\left(\mathcal{P}_{k}\right)$. (b) Next we consider the case $\mathcal{P}=(\mathcal{W})$ and let $H \cong \mathrm{GL}_{n-1}(q)$ be a standard subgroup of $G$, so that $H \cap S \cong \mathrm{SL}_{n-1}(q)$ is also a standard subgroup of $S$. We already mentioned that Weil representations of $H$ restrict irreducibly to Weil representations of $H \cap S$. In particular, if $\Phi$ has property $(\mathcal{W})$ then so does $\left.\Phi\right|_{S}$. Similarly, as the composition factors of $\Psi$ are among the composition factors of $\left.\left(\Psi^{G}\right)\right|_{S}$, if $\Psi^{G}$ has property $(\mathcal{W})$ then so does $\Psi$.

Conversely, observe that

$$
\left(\tau_{m, q}^{i}\right)^{G}=\sum_{j=0}^{q-2} \tau_{m, q}^{i, j} .
$$

Hence, if $\Theta$ is a Weil representation of $\operatorname{SL}_{m}(q)$ and $m \geq 3$, then every composition factor of $\Theta^{\mathrm{GL}_{m}(q)}$ is a Weil representation of $\mathrm{GL}_{m}(q)$ or a representation of degree 1. Moreover, we can find a cyclic subgroup $C \cong C_{q-1}$ of $H$ such that $G=S \rtimes C$ and 
$H=(H \cap S) \rtimes C$. It follows that if $\Psi$ has property $(\mathcal{W})$ then so does $\Psi^{G}$. Finally, as the composition factors of $\Phi$ are among the composition factors of $\left(\left.\Phi\right|_{S}\right)^{G}$, if $\left.\Phi\right|_{S}$ has property $(\mathcal{W})$ then so do $\left(\left.\Phi\right|_{S}\right)^{G}$ and $\Phi$.

Corollary 2.6. Let $G=\mathrm{GL}_{n}(q)$ or $\mathrm{SL}_{n}(q)$ and let $\Phi$ be an $\mathbb{F} G$-representation.

(i) If $\Phi$ possesses property $(\mathcal{W})$ and $n \geq 5$, then $\Phi$ has property $(\mathcal{Z})$.

(ii) If $\Phi$ has property $\left(\mathcal{P}_{k}\right)$ for some $2 \leq k \leq n-2$ and $n \geq 4$, then the Brauer character $\varphi$ of $\Phi$ satisfies $\varphi[1]=0$.

(iii) Suppose that either the assumption of (i) or of (ii) holds. If $P_{1}$ is the stabilizer in $G$ of a 1-space of the natural module $W=\mathbb{F}_{q}^{n}$ of $G$ and $V$ is an $\mathbb{F} G$-module affording $\Phi$, then $\boldsymbol{C}_{V}\left(Q_{1}\right) \neq 0$ for $Q_{1}:=\boldsymbol{O}_{p}\left(P_{1}\right)$.

Proof. (i) Suppose that $n \geq 5$ and $\Phi$ has property $(\mathcal{W})$. Then we can choose $\boldsymbol{x}, \boldsymbol{y}, \boldsymbol{z}$ from a standard subgroup $H=\mathrm{GL}_{n-1}(q)$ or $\mathrm{SL}_{n-1}(q)$ of $G$. By Lemma 2.4, $\left.\Phi\right|_{H}$ has property $(\mathcal{Z})$, and so does $\Phi$.

(ii) Suppose now that $n \geq 4$ and $\Phi$ has property $\left(\mathcal{P}_{k}\right)$. Then we can choose $\boldsymbol{x}, \boldsymbol{y}, \boldsymbol{z}$ from a standard subgroup $H=\mathrm{GL}_{4}(q)$ or $\mathrm{SL}_{4}(q)$ of $G$. First we consider the case $n=4$, so that $k=2$, and let $\chi:=\tau_{4, q}^{0,0} \in \operatorname{Irr}(H)$; in particular, $\chi(1)=\left(q^{4}-q\right) /(q-1)$. By Lemma 2.4, $\chi$ has property $(\mathcal{Z})$. On the other hand, in the notation of the proof of Corollary 2.3, it follows from the arguments in that proof that

$$
\left.\chi\right|_{Q}=\sum_{\lambda \in \mathcal{O}_{1}} \lambda+(2 q+1) \cdot 1_{Q} .
$$

Hence, choosing $\boldsymbol{x}, \boldsymbol{y} \in Q$, we see that

$$
\alpha[1]=\gamma[1]=0
$$

for $\alpha:=1_{Q}$ and $\gamma:=\sum_{\lambda \in \mathcal{O}_{1}} \lambda$. Also note that $P$ always acts transitively on $\mathcal{O}_{1}$, no matter if $H=\mathrm{GL}_{4}(q)$ or $\mathrm{SL}_{4}(q)$. It follows that $\left.\varphi\right|_{Q}=a \alpha+b \gamma$ for some integers $a, b \geq 0$, and so (2-2) implies that $\varphi[1]=0$.

Now we consider the case $n \geq 5$. If $G=\mathrm{GL}_{n}(q)$, then by Proposition 2.1(i), $\left.\Phi\right|_{H}$ has property $\left(\mathcal{P}_{2}\right)$ and so $\varphi[1]=0$ by the case $n=4$. Suppose now that $G=\operatorname{SL}_{n}(q)$ and set $M=\mathrm{GL}_{n}(q) \geq G$. We can choose a standard subgroup $L \cong \mathrm{GL}_{4}(q)$ in $M$ such that $L \cap G=H \cong \mathrm{SL}_{4}(q)$. By Proposition 2.5(ii) applied to $(M, G), \Phi^{M}$ has property $\left(\mathcal{P}_{k}\right)$, and so $\left.\left(\Phi^{M}\right)\right|_{L}$ has property $\left(\mathcal{P}_{2}\right)$ by Proposition $2.1(\mathrm{i})$. But then $\left.\left(\Phi^{M}\right)\right|_{H}$ has property $\left(\mathcal{P}_{2}\right)$ by Proposition $2.5(\mathrm{i})$. As we can find a cyclic subgroup $C \cong C_{q-1}$ of $L$ such that $L=H \rtimes C$ and $M=G \rtimes C$, we see that $\left.\left(\Phi^{M}\right)\right|_{H}=\left.\left(\left(\left.\Phi\right|_{H}\right)^{L}\right)\right|_{H}$. We can now conclude that $\Phi_{H}$ has property $\left(\mathcal{P}_{2}\right)$ and so $\varphi[1]=0$ again by the case $n=4$.

(iii) By the results of (i) and (ii), we may assume that $\varphi[1]=0$ for the Brauer character $\varphi$ of $\Phi$. Assume the contrary that $C_{V}\left(Q_{1}\right)=0$. Note that $P_{1}$ acts 
transitively on $\operatorname{IBr}\left(Q_{1}\right) \backslash\left\{1_{Q_{1}}\right\}$. It follows that we can write

$$
\varphi_{Q_{1}}=c \sum_{1_{Q_{1}} \neq \lambda \in \operatorname{IBr}\left(Q_{1}\right)} \lambda
$$

for some integer $c>0$. In particular, taking $\boldsymbol{x} \in Q_{1}$ we get

$$
\varphi(1)=c\left(q^{n-1}-1\right), \quad \varphi(\boldsymbol{x})=-c,
$$

and so the relation $\varphi[1]=0$ implies that

$$
\varphi(\boldsymbol{y})=-c\left(q^{n-2}+1\right) .
$$

On the other hand, as $n \geq 4$, we can choose an $\operatorname{SL}_{n}(q)$-conjugate $\boldsymbol{y}_{1} \in P_{1}$ of $\boldsymbol{y}$ that projects onto a transvection in $\mathrm{GL}_{n-1}(q)$ under the embedding

$$
P_{1} / Q_{1} \hookrightarrow \mathrm{GL}_{1}(q) \times \mathrm{GL}_{n-1}(q) .
$$

Such an element $y_{1}$ acts on $\operatorname{IBr}\left(Q_{1}\right) \backslash\left\{1_{Q_{1}}\right\}$ with exactly $q^{n-2}-1$ fixed points. Coupled with (2-3), this implies that

$$
|\varphi(\boldsymbol{y})|=\left|\varphi\left(\boldsymbol{y}_{1}\right)\right| \leq\left(q^{n-2}-1\right) c,
$$

contradicting (2-4).

\section{The general linear groups}

For $G=\mathrm{GL}_{n}(q)$ and $V$ an irreducible $\mathbb{F} G$-module, we will use James' parametrization [1986]

$$
V=\left(D\left(s_{1}, \lambda_{1}\right) \circ D\left(s_{2}, \lambda_{2}\right) \circ \cdots \circ D\left(s_{t}, \lambda_{t}\right)\right) \uparrow G
$$

for $V$ as given in [Guralnick and Tiep 1999, Proposition 2.4]. Here, $s_{i} \in \overline{\mathbb{F}}^{\times}$has degree $d_{i}$ over $\mathbb{F}_{q}$ and is $\ell$-regular, $\lambda_{i} \vdash k_{i}$, and $n=\sum_{i=1}^{t} k_{i} d_{i}$. Moreover, for any $i \neq j, s_{i}$ and $s_{j}$ do not have the same minimal polynomial over $\mathbb{F}_{q}$. Each $D\left(s_{i}, \lambda_{i}\right)$ is an irreducible module for $\mathrm{GL}_{k_{i} d_{i}}(q)$, and if $t>1$ then $V$ is Harish-Chandra-induced from the Levi subgroup

$$
L=\mathrm{GL}_{k_{1} d_{1}}(q) \times \mathrm{GL}_{k_{2} d_{2}}(q) \times \cdots \times \mathrm{GL}_{k_{t} s_{t}}(q)
$$

of a certain parabolic subgroup $P=Q L$ of $G$. Namely, consider

$$
D\left(s_{1}, \lambda_{1}\right) \otimes D\left(s_{2}, \lambda_{2}\right) \otimes \cdots \otimes D\left(s_{t}, \lambda_{t}\right)
$$

as an irreducible $L$-module, inflate it to an irreducible $\mathbb{F} P$-module $U$, and then induce to $G$ to get $V: V=U^{G}$. Note that the Harish-Chandra induction is commutative (with respect to the factors $\left.D\left(s_{i}, \lambda_{i}\right)\right)$ and transitive. Also, note that the Weil modules of $G$ are precisely $D(a,(n-1,1))$ and $(D(a,(n-1)) \circ D(b,(1))) \uparrow G$, with $a, b \in \mathbb{F}_{q}^{\times}$being $\ell$-regular and $a \neq b$. For the irreducible complex $G$-modules, we will instead use the notation

$$
\left(S\left(s_{1}, \lambda_{1}\right) \circ S\left(s_{2}, \lambda_{2}\right) \circ \cdots \circ S\left(s_{t}, \lambda_{t}\right)\right) \uparrow G .
$$


Proposition 3.1. Theorem $B$ holds for $G=\mathrm{GL}_{4}(q)$.

Proof. The implication "(i) $\Rightarrow$ (ii)" follows from Corollary 2.3. For the other implication, let $\Phi$ be an irreducible $\mathbb{E} G$-representation possessing property $\left(\mathcal{P}_{2}\right)$ and let $\varphi$ denote its Brauer character. By Corollary 2.6(ii)-(iii), we have $\varphi[1]=0$ and $\boldsymbol{C}_{V}\left(Q_{1}\right) \neq 0$, if $V$ is an $\mathbb{F} G$-module affording $\Phi$.

(i) First we consider the case $\ell=0$, or more generally, $\varphi$ lifts to a complex character of $G$. Using the character table of $G$ given, e.g., in [Geck et al. 1996], one can check that the relation $\varphi[1]=0$ implies that $\varphi$ is a Weil character. (Note that the character table of $G$ was first determined in [Steinberg 1951].)

(ii) From now on we may assume that $p \neq \ell|| G \mid$ and that $\varphi$ does not lift to a complex character. We use the label for $V$ given in (3-1). Since any irreducible $\mathbb{F} X$-module of $X \in\left\{\mathrm{GL}_{1}(q), \mathrm{GL}_{2}(q)\right\}$ lifts to a complex module, we see that $D\left(s_{i}, \lambda_{i}\right)$ lifts to a complex $\mathrm{GL}_{k_{i} d_{i}}(q)$-module if $k_{i} d_{i} \leq 2$. The same also happens if $\lambda_{i}=\left(k_{i}\right)$; see [Guralnick and Tiep 1999, Corollary 2.6]. Hence, the condition that $V$ does not lift to a complex module implies that one of the following cases must occur for $V$ as labeled in (3-1):

(a) $t=1, V=D(s, \lambda)$.

(b) $t=2, V=(D(a, \lambda) \circ D(b,(1))) \uparrow G, \operatorname{deg}(a)=\operatorname{deg}(b)=1, \lambda \vdash 3$, and $a \neq b$.

Let $e$ be the smallest positive integer such that $\ell \mid\left(1+q+q^{2}+\cdots+q^{e-1}\right)$.

(iii) Suppose we are in case (a). Then, by [Kleshchev and Tiep 2010, Theorem 5.4], $C_{V}\left(Q_{1}\right)=0$ whenever $\operatorname{deg}(s)>1$. Hence we must have that $\operatorname{deg}(s)=1$. By [Guralnick and Tiep 1999, Lemma 2.9] without loss we may assume that $s=1$, i.e., $\Phi$ is a unipotent representation. Note that $\Phi$ has degree 1 if $\lambda=(4)$ and is a Weil representation if $\lambda=(3,1)$. Now we let $\chi_{j}, 1 \leq j \leq 5$, denote the complex unipotent character of $G$ labeled by $(4),(3,1),(2,2),\left(2,1^{2}\right)$, and $\left(1^{4}\right)$, respectively. Similarly, we let $\varphi_{j}, 1 \leq j \leq 5$, denote the Brauer unipotent character of $G$ labeled by $(4),(3,1),(2,2),\left(2,1^{2}\right)$, and $\left(1^{4}\right)$, respectively. Then

$$
\chi_{1}[1]=\chi_{2}[1]=\varphi_{1}[1]=\varphi_{2}[1]=0, \quad \chi_{3}[1]=q^{4}, \quad \chi_{4}[1]=q^{5}, \quad \chi_{5}[1]=q^{6} .
$$

Recall that we use the notation $\chi^{\circ}$ to denote the restriction of a complex character $\chi$ to the set of $\ell^{\prime}$-elements of $G$, and that $\varphi[1]=0$.

By the results of [James 1990], there are integers $x_{1}, x_{2}$ such that

$$
\chi_{3}^{\circ}=\varphi_{3}+x_{1} \varphi_{1}+x_{2} \varphi_{2} .
$$

It follows by (3-2) that $\varphi_{3}[1]=\chi_{3}[1]=q^{4}$; in particular, $\lambda \neq(2,2)$.

Next, by [Guralnick and Tiep 1999, Proposition 3.1], there are nonnegative integers $y_{1}, y_{2}, y_{3}$ such that $y_{3} \leq 1$ and

$$
\chi_{4}^{\circ}=\varphi_{4}+y_{1} \varphi_{1}+y_{2} \varphi_{2}+y_{3} \varphi_{3} .
$$


It follows by (3-2) that

$$
\varphi_{4}[1]=\chi_{4}[1]-y_{3} \varphi_{3}[1]=q^{4}\left(q-y_{3}\right)>0 ;
$$

in particular, $\lambda \neq\left(2,1^{2}\right)$.

Finally, let $\lambda=\left(1^{4}\right)$, i.e., $\varphi=\varphi_{5}$. Then $\lambda$ is 2 -divisible and 4-divisible in the sense of [Kleshchev and Tiep 2010, Definition 4.3]. Since $C_{V}\left(Q_{1}\right) \neq 0$, it follows by [loc. cit., Theorem 5.4] that $e \neq 2,4$. If $e=3$, then by [James 1990], we have

$$
\chi_{5}^{\circ}=\varphi+\varphi_{3},
$$

and so $\chi_{5}[1]=\varphi_{3}[1]=q^{4}$, contrary to (3-2). Thus we must have $e \geq 5>n$, and so $\varphi_{5}=\chi_{5}^{\circ}$ by [James 1990, Theorem 6.4], whence $\chi_{5}[1]=\varphi[1]=0$, again a contradiction.

(iv) Suppose we are in case (b). By [Guralnick and Tiep 1999, Lemma 2.9], we may assume that $a=1$. Let $\rho_{1}, \rho_{2}$, and $\rho_{3}$ denote the (ordinary) characters of the irreducible $\mathbb{C} G$-modules $(S(1, \mu) \circ S(b,(1))) \uparrow G$, with $\mu=(3),(2,1)$, and $\left(1^{3}\right)$, respectively. Similarly, let $\psi_{1}, \psi_{2}$, and $\psi_{3}$ denote the Brauer characters of the irreducible $\mathbb{F} G$-modules $(D(1, \mu) \circ D(b,(1))) \uparrow G$, with $\mu=(3),(2,1)$, and $\left(1^{3}\right)$, respectively. Using [Geck et al. 1996], we can compute

$$
\rho_{1}[1]=0, \quad \rho_{2}[1]=q^{4}(q+1), \quad \rho_{3}[1]=q^{5}(q+1) .
$$

Note that $\psi_{1}$ is a Weil character, and so $\psi_{1}[1]=0$. Using the decomposition matrix for $\mathrm{GL}_{3}(q)$ [James 1990], we get a nonnegative integer $x$ such that

$$
\rho_{2}^{\circ}=\psi_{2}+x \psi_{1} \text {. }
$$

It follows by (3-3) that $\psi_{2}[1]=\rho_{2}[1]=q^{4}(q+1)$. Furthermore, there are integers $0 \leq y, z \leq 1$ such that

$$
\rho_{3}^{\circ}=\psi_{3}+y \psi_{1}+z \psi_{2} \text {. }
$$

It follows by (3-3) that

$$
\psi_{3}[1]=\rho_{3}[1]-z \psi_{2}[1] \geq q^{4}\left(q^{2}-1\right) .
$$

We have therefore shown that $\varphi=\psi_{1}$, a Weil character.

Proposition 3.2. Let $G=\mathrm{GL}_{n}(q)$ with $n \geq 5$ and let $V$ be an irreducible $\mathbb{F} G$-module. Suppose that $V$ has property $(\mathcal{W})$. Then in the label (3-1) for $V, \operatorname{deg}\left(s_{i}\right)=1$ for all $i$. Furthermore, either $V$ is a Weil module, or $t=1$.

Proof. (i) First we consider the case $t=1$. By Corollary 2.6, $\boldsymbol{C}_{V}\left(Q_{1}\right) \neq 0$, whence $\operatorname{deg}\left(s_{1}\right)=1$ by [Kleshchev and Tiep 2010, Theorem 5.4].

(ii) From now on we may assume that $t \geq 2$, so that $V$ is Harish-Chandrainduced. Next we show that $\operatorname{deg}\left(s_{i}\right)=1$ for all $i$. Assume for instance that 
$d_{1}=\operatorname{deg}\left(s_{1}\right)>1$. Then we consider a standard subgroup $H=\operatorname{GL}_{m}(q)$ of $G$ with $2 \leq m:=n-k_{t} d_{t} \leq n-1$. Property $(\mathcal{W})$ implies that all composition factors of $\left.V\right|_{H}$ are Weil modules or of dimension 1. We can find a parabolic subgroup $P=Q L$ with Levi subgroup $L=H \times \mathrm{GL}_{k_{t} d_{t}}(q)$. Then note that $V$ can be obtained by inflating the irreducible $\mathbb{F} L$-module $A \otimes D\left(s_{t}, \lambda_{t}\right)$ to $P$, where

$$
A:=\left(D\left(s_{1}, \lambda_{1}\right) \circ D\left(s_{2}, \lambda_{2}\right) \circ \cdots \circ D\left(s_{t-1}, \lambda_{t-1}\right)\right) \uparrow H,
$$

and then induce to $G$. In particular, $A$ is a simple submodule of $\left.V\right|_{H}$, and clearly $A$ is not a Weil module as $\operatorname{deg}\left(s_{1}\right)>1$. Suppose that $\operatorname{dim}(A)=1$. Since $\operatorname{deg}\left(s_{1}\right)>1$, we must have that $(m, q)=(2,2), \operatorname{deg}\left(s_{1}\right)=2, \lambda_{1}=(1)$, and $t=2$. Applying property $(\mathcal{W})$ to a standard subgroup $\mathrm{GL}_{3}(2)$ containing $H=\mathrm{GL}_{2}(2)$ (as its standard subgroup) and then restricting further down to $H$, we see, however, that $A=\operatorname{deg}\left(s_{1},(1)\right)$ cannot occur in $\left.V\right|_{H}$, a contradiction.

(iii) Here we show that one of the following holds:

(a) $t=3, \lambda_{i}=\left(k_{i}\right)$ for all $i$, and $\left\{k_{1}, k_{2}, k_{3}\right\}=\{n-2,1,1\}$.

(b) $t=2$ and $\lambda_{i} \in\left\{\left(k_{i}\right),\left(k_{i}-1,1\right)\right\}$ for all $i$.

The arguments in (ii) show that $A$ is a simple submodule of $\left.V\right|_{H}$. Again by property $(\mathcal{W}), A$ is either a Weil module or of dimension 1 . First we consider the case $t \geq 3$. Then note that $\operatorname{dim}(A)$ is at least the index of a proper parabolic subgroup of $H$ and so can never be equal to 1 . Thus $A$ is a Weil module. The identification of Weil modules among the ones labeled in (3-1) now implies that in fact $t=3$, $\lambda_{i}=\left(k_{i}\right)$ for all $i=1,2$, and $1 \in\left\{k_{1}, k_{2}\right\}$. Interchanging $k_{3}$ with $k_{1}$ or $k_{2}$ in the above construction and noting that $n \geq 5$, we then get $k_{3}=1$ and $\lambda_{3}=(1)$ as well.

Suppose now that $t=2$. The claim is obvious if $k_{i} \leq 2$, so we consider the case where $k_{1} \geq 3$, say. Then $A=D\left(s_{1}, \lambda_{1}\right)$ is a Weil module of $\operatorname{GL}_{k_{1}}(q)$ or of dimension 1. It follows that $\lambda_{1} \in\left\{\left(k_{1}\right),\left(k_{1}-1,1\right)\right\}$, as stated.

(iv) Abusing the notation, now we use $H$ to denote the standard subgroup

$$
H=\operatorname{Stab}_{G}\left(\left\langle e_{1}, e_{2}, \ldots, e_{n-1}\right\rangle_{\mathbb{F}_{q}}, e_{n}\right) \cong \operatorname{GL}_{n-1}(q),
$$

and $P=Q \rtimes L$ to denote the parabolic subgroup

$$
P=\operatorname{Stab}_{G}\left(\left\langle e_{1}, \ldots, e_{k}\right\rangle_{\mathbb{F}_{q}}\right),
$$

where $k:=k_{1}$ and

$$
L=\operatorname{Stab}_{G}\left(\left\langle e_{1}, \ldots, e_{k}\right\rangle_{\mathbb{F}_{q}},\left\langle e_{k+1}, \ldots, e_{n}\right\rangle_{\mathbb{F}_{q}}\right) .
$$

Then we can obtain $V$ by inflating the irreducible $\mathbb{F} L$-module $B$, where

$$
B:=D\left(s_{1}, \lambda_{1}\right) \otimes\left(D\left(s_{2}, \lambda_{2}\right) \circ \cdots \circ D\left(s_{t}, \lambda_{t}\right)\right) \uparrow \mathrm{GL}_{n-k}(q),
$$


to an irreducible $\mathbb{F} P$-module $U$ and then induce to $G$. Note that $P_{H}=Q_{H} \rtimes L_{H}$ is also a parabolic subgroup of $H$, where

$$
P_{H}:=P \cap H, \quad Q_{H}:=Q \cap H, \quad L_{H}:=L \cap H .
$$

Mackey's formula implies that $\left.V\right|_{H}$ contains a subquotient $V^{\prime}:=\left(U^{\prime}\right)^{H}$, where $U^{\prime}:=\left.U\right|_{P_{H}}$. But note that $Q_{H} \leq Q$ acts trivially on $U$, so in fact $V^{\prime}$ is HarishChandra-induced from the $\mathbb{F} L_{H}$-module $B^{\prime}$, where $B^{\prime}:=\left.B\right|_{L_{H}}$.

(v) Now we can complete the case $t=3$. As shown in (iii), in this case we have that $\lambda_{i}=\left(k_{i}\right)$ for all $i$ and we may furthermore assume that $\left(k_{1}, k_{2}, k_{3}\right)=(1,1, n-2)$. Repeating the argument in (iv) and using the notation therein, we see that $B^{\prime}$ contains a simple subquotient isomorphic to

$$
D\left(s_{1},(1)\right) \otimes\left(D\left(s_{2},(1)\right) \circ D\left(s_{3},(n-3)\right)\right) \uparrow \mathrm{GL}_{n-2}(q) .
$$

It follows that $\left.V\right|_{H}$ contains a subquotient isomorphic to

$$
\left(D\left(s_{1},(1)\right) \circ D\left(s_{2},(1)\right) \circ D\left(s_{3},(n-3)\right)\right) \uparrow H,
$$

which is irreducible, but not a Weil module nor of dimension 1 . This contradiction shows that the case $t=3$ is impossible.

(vi) Finally, we consider the case $t=2$. As shown in (iii), $\lambda_{i} \in\left\{\left(k_{i}\right),\left(k_{i}-1,1\right)\right\}$; also, recall that $n=k_{1}+k_{2} \geq 5$. Suppose first that $k_{2} \geq k_{1} \geq 2$. Then, in the notation of (iv), we see that $B=D\left(s_{1}, \lambda_{1}\right) \otimes D\left(s_{2}, \lambda_{2}\right)$ and so $B^{\prime}$ contains a simple subquotient isomorphic to $D\left(s_{1}, \lambda_{1}\right) \otimes D\left(s_{2}, \mu\right)$ with $\mu \in\left\{\left(k_{2}-1\right),\left(k_{2}-2,1\right)\right\}$. It follows that $\left.V\right|_{H}$ contains a subquotient isomorphic to

$$
\left(D\left(s_{1}, \lambda_{1}\right) \circ D\left(s_{2}, \mu\right)\right) \uparrow H,
$$

which is irreducible, but not a Weil module nor of dimension 1, a contradiction.

Hence we may assume that $\left(k_{1}, k_{2}\right)=(1, n-1)$. If $\lambda_{2}=(n-1)$, then $V$ is a Weil module. Assume that $\lambda_{2}=(n-2,1)$. Then, in the notation of (iv), we see that $B=D\left(s_{1}, \lambda_{1}\right) \otimes D\left(s_{2},(n-2,1)\right)$ and so $B^{\prime}$ contains a simple subquotient isomorphic to $D\left(s_{1}, \lambda_{1}\right) \otimes D\left(s_{2},(n-3,1)\right)$. It follows that $\left.V\right|_{H}$ contains a subquotient isomorphic to

$$
\left(D\left(s_{1},(1)\right) \circ D\left(s_{2},(n-3,1)\right)\right) \uparrow H,
$$

which is irreducible, but not a Weil module nor of dimension 1, again contradicting property $(\mathcal{W})$.

Proposition 3.3. Let $G=\mathrm{GL}_{n}(q)$ with $n \geq 5$ and let $V$ be an irreducible $\mathbb{F} G$-module. Suppose that $V$ has property $(\mathcal{W})$. Then either $V$ is a Weil module, or $\operatorname{dim} V=1$.

Proof. (i) Consider the label (3-1) for $V$. By Proposition 3.2 and [Guralnick and Tiep 1999, Lemma 2.9], we may assume that $V=D(1, \lambda)$, a unipotent representation; furthermore, $C_{V}\left(Q_{1}\right) \neq 0$ by Corollary 2.6. Now $V$ is a subquotient of the 
reduction modulo $\ell$ of the unipotent $\mathbb{C} G$-module $S(1, \lambda)$. We can find a standard subgroup $H=\mathrm{GL}_{n-1}(q)$ of $G$ as a direct factor of the Levi subgroup $L_{1}=$ $H \times \mathrm{GL}_{1}(q)$ of $P_{1}=Q_{1} L_{1}$. By the Howlett-Lehrer comparison theorem [1983, Theorem 5.9], the Harish-Chandra restriction ${ }^{*} R_{L_{1}}^{G}$ of unipotent characters of $G$ can be computed inside the Weyl group $S_{n}$ of $G$, and it is similar for the HarishChandra induction $R_{L_{1}}^{G}$. For brevity, we denote the character of $S(1, \lambda)$ by $\chi^{\lambda}$ and the Brauer character of $D(1, \lambda)$ by $\varphi^{\lambda}$, with similar notation for other partitions. In this notation, ${ }^{*} R_{L_{1}}^{G}\left(\chi^{\lambda}\right)$ is the sum of unipotent characters $\chi^{\mu}$ of $L_{1}$ labeled by $\mu \vdash(n-1)$, where the Young diagram $Y(\mu)$ of $\mu$ is obtained from the Young diagram $Y(\lambda)$ of $\lambda$ by removing one removable node. For instance,

$$
\begin{aligned}
{ }^{*} R_{L_{1}}^{G}\left(\chi^{(n)}\right) & =\chi^{(n-1)}, \\
{ }^{*} R_{L_{1}}^{G}\left(\chi^{(n-1,1)}\right) & =\chi^{(n-1)}+\chi^{(n-2,1)}, \\
{ }^{*} R_{L_{1}}^{G}\left(\chi^{(n-2,2)}\right) & =\chi^{(n-3,2)}+\chi^{(n-2,1)}, \\
{ }^{*} R_{L_{1}}^{G}\left(\chi^{\left(n-2,1^{2}\right)}\right) & =\chi^{\left(n-3,1^{2}\right)}+\chi^{(n-2,1)} .
\end{aligned}
$$

It is similar for the Harish-Chandra induction; in particular,

$$
\begin{aligned}
R_{L_{1}}^{G}\left(\chi^{(n-1)}\right) & =\chi^{(n)}+\chi^{(n-1,1)}, \\
R_{L_{1}}^{G}\left(\chi^{(n-2,1)}\right) & =\chi^{(n-1,1)}+\chi^{(n-2,2)}+\chi^{\left(n-2,1^{2}\right)} .
\end{aligned}
$$

Let $\psi$ be the Brauer character of a simple submodule of the $L_{1}$-module $C_{V}\left(Q_{1}\right)$. Then $\psi$ is an irreducible constituent of ${ }^{*} R_{L_{1}}^{G}\left(\varphi^{\lambda}\right)$, the Brauer $L_{1}$-character of $C_{V}\left(Q_{1}\right)$. The above arguments show that $\psi$ is an irreducible constituent of $\left(\chi^{\mu}\right)^{\circ}$ for some $\mu \vdash(n-1)$, whence $\psi=\varphi^{v}$ for some $v \vdash(n-1)$. On the other hand, property $(\mathcal{W})$ implies that $\psi$ is a Weil character (while restricted to $H$ ), or has degree 1. As $n \geq 5$, it follows that $v=(n-1)$ or $(n-2,1)$. By Frobenius' reciprocity, $\varphi^{\lambda}$ is an irreducible constituent of $R_{L_{1}}^{G}\left(\varphi^{v}\right)$, and so of $\left(R_{L_{1}}^{G}\left(\chi^{v}\right)\right)^{\circ}$ as well. Restricting (3-5) to $\ell^{\prime}$-elements, we see by [Guralnick and Tiep 1999, Proposition 3.1] that $\lambda$ is $(n),(n-1,1),(n-2,2)$, or $\left(n-2,1^{2}\right)$. The first possibility leads to the principal character, and the second one yields a Weil character.

(ii) Here we consider the case $\lambda=(n-2,2)$. Then applying [loc. cit., Proposition 3.1] to $G$, we can write

$$
\left(\chi^{\lambda}\right)^{\circ}=\varphi^{\lambda}+x_{1}\left(\chi^{(n)}\right)^{\circ}+x_{2}\left(\chi^{(n-1,1)}\right)^{\circ}
$$

for some integers $x_{1}, x_{2}$. It follows by (3-4) that

$$
{ }^{*} R_{L_{1}}^{G}\left(\varphi^{\lambda}\right)=\left(\chi^{(n-3,2)}+\left(1-x_{2}\right) \chi^{(n-2,1)}-\left(x_{1}+x_{2}\right) \chi^{(n-1)}\right)^{\circ} .
$$

Applying [loc. cit., Proposition 3.1] to $H$, we then get

$$
{ }^{*} R_{L_{1}}^{G}\left(\varphi^{\lambda}\right)=\varphi^{(n-3,2)}+x_{1}^{\prime} \varphi^{(n-1)}+x_{2}^{\prime} \varphi^{(n-2,1)}
$$


for some integers $x_{1}^{\prime}, x_{2}^{\prime}$. By the linear independence of irreducible Brauer characters, $\varphi^{(n-3,2)}$ is an irreducible constituent of $\left.\left(\varphi^{\lambda}\right)\right|_{H}$, contradicting $(\mathcal{W})$.

(iii) Finally, assume that $\lambda=\left(n-2,1^{2}\right)$. Then applying [loc. cit., Proposition 3.1] to $G$, we have

$$
\left(\chi^{\lambda}\right)^{\circ}=\varphi^{\lambda}+y_{1}\left(\chi^{(n)}\right)^{\circ}+y_{2}\left(\chi^{(n-1,1)}\right)^{\circ}+y_{3}\left(\chi^{(n-2,2)}\right)^{\circ}
$$

for some integers $y_{1}, y_{2}, y_{3}$. It follows by (3-4) that

$$
{ }^{*} R_{L_{1}}^{G}\left(\varphi^{\lambda}\right)=\left(\chi^{\left(n-3,1^{2}\right)}-y_{3} \chi^{(n-3,2)}+\left(1-y_{2}-y_{3}\right) \chi^{(n-2,1)}-\left(y_{1}+y_{2}\right) \chi^{(n-1)}\right)^{\circ} .
$$

Applying [loc. cit., Proposition 3.1] to $H$, we then get

$$
{ }^{*} R_{L_{1}}^{G}\left(\varphi^{\lambda}\right)=\varphi^{\left(n-3,1^{2}\right)}+y_{1}^{\prime} \varphi^{(n-1)}+y_{2}^{\prime} \varphi^{(n-2,1)}+y_{3}^{\prime} \varphi^{(n-2,2)}
$$

for some integers $y_{1}^{\prime}, y_{2}^{\prime}, y_{3}^{\prime}$. By linear independence of irreducible Brauer characters, $\varphi^{\left(n-3,1^{2}\right)}$ is an irreducible constituent of $\left.\left(\varphi^{\lambda}\right)\right|_{H}$, again contradicting $(\mathcal{W})$.

We note (without giving proof, since we do not need it subsequently) that property $(\mathcal{Z})$ can also be used to characterize Weil representations of $\mathrm{GL}_{n}(q)$ with $n \geq 5$.

Proposition 3.4. Theorem $A$ holds for $G=\mathrm{GL}_{n}(q)$ with $n \geq 5$.

Proof. The implication "(i) $\Rightarrow$ (ii)" follows from Corollary 2.3. In fact, by applying Corollary 2.3 successively, we see that (i) also implies (iii) and (iv). Also, note that (iv) obviously implies (iii), and (ii) implies (i) by Proposition 3.3.

It remains to show that (iii) implies (i). We proceed by induction on $n \geq 5$, with the induction base $n=5$ (so that $m=4$ ) already established in Proposition 3.3. For the induction step $n \geq 6$, consider a chain of standard subgroups

$$
H=\mathrm{GL}_{m}(q) \leq L=\mathrm{GL}_{n-1}(q)<G=\mathrm{GL}_{n}(q) .
$$

Let $\Psi$ be any composition factor of $\left.\Phi\right|_{L}$. According to (iii), every composition factor of $\left.\Psi\right|_{H}$ is either a Weil representation or has dimension 1. Hence, by the induction hypothesis applied to $L$, we see that either $\Psi$ is a Weil representation or $\operatorname{deg} \Psi=1$. Thus $\Phi$ has property $(\mathcal{W})$, and so we are done by Proposition 3.3.

Proposition 3.5. Theorem $B$ holds for $G=\mathrm{GL}_{n}(q)$ with $n \geq 4$.

Proof. The implication "(i) $\Rightarrow$ (ii)" follows from Corollary 2.3. For the other implication, let $G=\mathrm{GL}_{n}(q)$ with $n \geq 4$ and let $\Phi$ be an irreducible $\mathbb{F} G$-representation with property $\left(\mathcal{P}_{k}\right)$ for some $2 \leq k \leq n-2$. By Proposition 3.1, we may assume that $n \geq 5$ and consider a standard subgroup $H \cong \mathrm{GL}_{4}(q)$ of $G$. By Corollary 2.2(i), every composition factor $\Psi$ of $\left.\Phi\right|_{H}$ has property $\left(\mathcal{P}_{2}\right)$. By Proposition 3.1, either $\Psi$ is a Weil representation or $\operatorname{deg} \Psi=1$. Thus $\Phi$ fulfills condition (iii) of Theorem A for $G$ and with $m=4$. Hence $\Phi$ is either a Weil representation or has degree 1 by Proposition 3.4. 


\section{The special linear groups}

Proof of Theorems A and B. By Propositions 3.4 and 3.5, it suffices to prove the theorems for $S=\operatorname{SL}_{n}(q)$. Also, by Corollary 2.3, it suffices to prove the implication "(ii) $\Rightarrow$ (i)" (as the implication "(iii) $\Rightarrow$ (i)" of Theorem A can then be proved using the same arguments as in the proof of Proposition 3.4). Let $\mathcal{P} \in\left\{\left(\mathcal{P}_{k}\right),(\mathcal{W})\right\}$ and let $U$ be an irreducible $\mathbb{F} S$-module with property $\mathcal{P}$. We consider $S$ as the derived subgroup of $G=\mathrm{GL}_{n}(q)$. By Proposition 2.5(ii), a simple submodule $V$ of $U^{G}$ has property $\mathcal{P}$. Applying Proposition 3.5 if $\mathcal{P}=\left(\mathcal{P}_{k}\right)$, respectively Proposition 3.4 if $\mathcal{P}=(\mathcal{W})$, to $V$, we see that $V$ is a Weil module or $\operatorname{dim} V=1$. As $U$ is an irreducible constituent of $\left.V\right|_{S}$, we conclude that $U$ is a Weil module or has dimension 1 .

We note that one could try to prove the complex case of Theorem A for $\operatorname{GL}_{n}(q)$ using the results of [Zelevinsky 1981] or [Thoma 1971]. We conclude by the following example showing that Weil representations of $\mathrm{GL}_{n}(q)$ and $\mathrm{SL}_{n}(q)$ do not admit a "middle-free" characterization in the spirit of [Guralnick et al. 2002, Corollary 12.4].

Example 4.1. Let $G=\mathrm{GL}_{n}(q)$ or $\mathrm{SL}_{n}(q)$ with $n \geq 3$. Consider the natural module $W=\left\langle e_{1}, \ldots, e_{n}\right\rangle_{\mathbb{F}_{q}}$ and let

$$
P=\operatorname{Stab}_{G}\left(\left\langle e_{1}\right\rangle_{\mathbb{F}_{q}},\left\langle e_{1}, \ldots, e_{n-1}\right\rangle_{\mathbb{F}_{q}}\right) .
$$

Note that $P=N_{G}(Z)$ for a long-root subgroup $Z$ of $G$. Also, we may assume that (a $G$-conjugate of) the long-root element $\boldsymbol{x}$ defined in Section 2 is contained in $Z$. Suppose that an irreducible $\mathbb{F} G$-module $V$ has no middle with respect to $Q=\boldsymbol{O}_{p}(P)$ as in [Guralnick et al. 2002, Corollary 12.4], i.e.,

$$
C_{V}(Z)=C_{V}(Q) \text {. }
$$

It follows that

$$
V=C_{V}(Q) \oplus[V, Z] .
$$

Let $\varphi$ denote the Brauer character of $V$. Then we have

$$
\varphi(\boldsymbol{x})=a-q^{n-2} b,
$$

where $\operatorname{dim} C_{V}(Q)=a$ and $\operatorname{dim}[V, Z]=q^{n-2}(q-1) b$. But note that $Q \backslash Z$ contains a $G$-conjugate $\boldsymbol{x}^{\prime}$ of $\boldsymbol{x}$, and

$$
\varphi\left(\boldsymbol{x}^{\prime}\right)=a .
$$

It follows that $b=0, V=C_{V}(Q), Q$ acts trivially on $V$, and so $\operatorname{dim} V=1$.

\section{Acknowledgements}

The author is grateful to Alexandre E. Zalesski for bringing the question of recognizing Weil representations of finite general and special linear groups to his attention. Part of the work was done while the author was visiting the Institute for 
Advanced Study, Princeton. It is a pleasure to thank Peter Sarnak and the Institute for generous hospitality and a stimulating environment. The author is also grateful to the referee for careful reading and helpful comments on the paper.

\section{References}

[Bolt et al. 1961] B. Bolt, T. G. Room, and G. E. Wall, "On the Clifford collineation, transform and similarity groups, I”, J. Austral. Math. Soc. 2:1 (1961), 60-79. MR 23 \#A3171 Zbl 0097.01702

[Brundan and Kleshchev 2000] J. Brundan and A. S. Kleshchev, "Lower bounds for degrees of irreducible Brauer characters of finite general linear groups", J. Algebra 223:2 (2000), 615-629. MR 2001f:20014 Zbl 0954.20022

[Conway et al. 1985] J. H. Conway, R. T. Curtis, S. P. Norton, R. A. Parker, and R. A. Wilson, Atlas of finite groups: maximal subgroups and ordinary characters for simple groups, Oxford University Press, Eynsham, 1985. MR 88g:20025 Zbl 0568.20001

[Dummigan 1996] N. Dummigan, "Symplectic group lattices as Mordell-Weil sublattices", J. Number Theory 61:2 (1996), 365-387. MR 98h:11088 Zbl 0869.11057

[Dummigan and Tiep 1999] N. Dummigan and P. H. Tiep, "Lower bounds for the minima of certain symplectic and unitary group lattices", Amer. J. Math. 121:4 (1999), 889-918. MR 2001a:11112 Zbl 0936.20004

[Geck et al. 1996] M. Geck, G. Hiss, F. Lübeck, G. Malle, and G. Pfeiffer, "CHEVIE: a system for computing and processing generic character tables", Appl. Algebra Engrg. Comm. Comput. 7:3 (1996), 175-210. MR 99m:20017 Zbl 0847.20006

[Gérardin 1977] P. Gérardin, "Weil representations associated to finite fields", J. Algebra 46:1 (1977), 54-101. MR 57 \#470 Zbl 0359.20008

[Gow 1989] R. Gow, "Even unimodular lattices associated with the Weil representation of the finite symplectic group”, J. Algebra 122:2 (1989), 510-519. MR 90f:20009 Zbl 0679.20039

[Gross 1990] B. H. Gross, "Group representations and lattices", J. Amer. Math. Soc. 3:4 (1990), 929-960. MR 92a:11077 Zbl 0745.11035

[Guralnick and Tiep 1999] R. M. Guralnick and P. H. Tiep, "Low-dimensional representations of special linear groups in cross characteristics", Proc. London Math. Soc. (3) 78:1 (1999), 116-138. MR 2000a:20016 Zbl 0974.20014

[Guralnick and Tiep 2004] R. M. Guralnick and P. H. Tiep, "Cross characteristic representations of even characteristic symplectic groups", Trans. Amer. Math. Soc. 356:12 (2004), 4969-5023. MR 2005j:20012 Zbl 1062.20013

[Guralnick et al. 2002] R. M. Guralnick, K. Magaard, J. Saxl, and P. H. Tiep, "Cross characteristic representations of symplectic and unitary groups", J. Algebra 257:2 (2002), 291-347. MR 2004b:20022 Zbl 1025.20002

[Guralnick et al. 2006] R. M. Guralnick, K. Magaard, J. Saxl, and P. H. Tiep, “Addendum: 'Cross characteristic representations of odd characteristic symplectic groups and unitary groups' [J. Algebra 257:2 (2002), 291-347]”, J. Algebra 299:1 (2006), 443-446. MR 2007e:20096 Zbl 1025.20002

[Hiss and Malle 2001] G. Hiss and G. Malle, "Low-dimensional representations of special unitary groups”, J. Algebra 236:2 (2001), 745-767. MR 2001m:20019 Zbl 0972.20027

[Howe 1973] R. E. Howe, "On the character of Weil's representation”, Trans. Amer. Math. Soc. 177 (1973), 287-298. MR 47 \#5180 Zbl 0263.22014

[Howlett and Lehrer 1983] R. B. Howlett and G. I. Lehrer, "Representations of generic algebras and finite groups of Lie type", Trans. Amer. Math. Soc. 280:2 (1983), 753-779. MR 85i:20044 Zbl 0537.20018 
[Isaacs 1973] I. M. Isaacs, "Characters of solvable and symplectic groups", Amer. J. Math. 95 (1973), 594-635. MR 48 \#11270 Zbl 0277.20008

[James 1986] G. James, "The irreducible representations of the finite general linear groups", Proc. London Math. Soc. (3) 52:2 (1986), 236-268. MR 87h:20028 Zbl 0587.20022

[James 1990] G. James, "The decomposition matrices of $\mathrm{GL}_{n}(q)$ for $n \leq 10$ ", Proc. London Math. Soc. (3) 60:2 (1990), 225-265. MR 91c:20024 Zbl 0760.20003

[Kleshchev and Tiep 2010] A. S. Kleshchev and P. H. Tiep, "Representations of the general linear groups which are irreducible over subgroups", Amer. J. Math. 132:2 (2010), 425-473. MR 2011k: 20091 Zbl 1211.20017

[Scharlau and Tiep 1997] R. Scharlau and P. H. Tiep, "Symplectic groups, symplectic spreads, codes, and unimodular lattices”, J. Algebra 194:1 (1997), 113-156. MR 98g:20075 Zbl 0887.20004

[Scharlau and Tiep 1999] R. Scharlau and P. H. Tiep, "Symplectic group lattices", Trans. Amer. Math. Soc. 351:5 (1999), 2101-2139. MR 99k:20010 Zbl 0941.20003

[Seitz 1975] G. M. Seitz, "Some representations of classical groups”, J. London Math. Soc. (2) 10 (1975), 115-120. MR 51 \#5789 Zbl 0333.20039

[Steinberg 1951] R. Steinberg, "The representations of GL(3, $q)$, GL(4, $q)$, PGL(3, $q)$, and PGL(4, q)", Canadian J. Math. 3 (1951), 225-235. MR 13,10e Zbl 0042.25602

[Thoma 1971] E. Thoma, "Die Einschränkung der Charaktere von GL $(n, q)$ auf GL $(n-1, q)$ ", Math. Z. 119 (1971), 321-338. MR 44 \#5388 Zbl 0205.32602

[Tiep 1997a] P. H. Tiep, "Globally irreducible representations of finite groups and integral lattices", Geom. Dedicata 64:1 (1997), 85-123. MR 98e:20011 Zbl 0867.20005

[Tiep 1997b] P. H. Tiep, "Weil representations as globally irreducible representations", Math. Nachr. 184 (1997), 313-327. MR 98b:20010 Zbl 0870.20007

[Tiep and Zalesski 1996] P. H. Tiep and A. E. Zalesski, "Minimal characters of the finite classical groups”, Comm. Algebra 24:6 (1996), 2093-2167. MR 97f:20018 Zbl 0901.20031

[Tiep and Zalesski 1997] P. H. Tiep and A. E. Zalesski, "Some characterizations of the Weil representations of the symplectic and unitary groups", J. Algebra 192:1 (1997), 130-165. MR 99d:20074 Zbl 0877.20030

[Ward 1972] H. N. Ward, "Representations of symplectic groups", J. Algebra 20 (1972), 182-195. MR 44 \#4116 Zbl 0239.20013

[Weil 1964] A. Weil, "Sur certains groupes d'opérateurs unitaires", Acta Math. 111 (1964), 143-211. MR 29\#2324 Zbl 0203.03305

[Zalesski 1988] A. E. Zalesski, "Eigenvalues of matrices of complex representations of finite groups of Lie type", pp. 206-218 in Algebra: some current trends (Varna, 1986), edited by L. L. Avramov and K. B. Tchakerian, Lecture Notes in Mathematics 1352, Springer, Berlin, 1988. MR 90d:20084 Zbl 0661.20032

[Zalesski $\geq 2015$ ] A. E. Zalesski, "Singer torus in irreducible representations of $G L_{n}(q)$ ", To appear in J. Group Theory.

[Zelevinsky 1981] A. V. Zelevinsky, Representations of finite classical groups: a Hopf algebra approach, Lecture Notes in Mathematics 869, Springer, Berlin, 1981. MR 83k:20017 Zbl 0465.20009

Received March 9, 2015. Revised July 22, 2015.

PhAM HuU TIEP

DEPARTMENT OF MATHEMATICS

UNIVERSITY OF ARIZONA

TUCSON, AZ 85721

UNITEd STATES

tiep@math.arizona.edu 


\title{
PACIFIC JOURNAL OF MATHEMATICS
}

\author{
msp.org/pjm
}

Founded in 1951 by E. F. Beckenbach (1906-1982) and F. Wolf (1904-1989)

\section{EDITORS}

Don Blasius (Managing Editor)

Department of Mathematics

University of California

Los Angeles, CA 90095-1555

blasius@math.ucla.edu

\author{
Paul Balmer \\ Department of Mathematics \\ University of California \\ Los Angeles, CA 90095-1555 \\ balmer@math.ucla.edu \\ Robert Finn \\ Department of Mathematics \\ Stanford University \\ Stanford, CA 94305-2125 \\ finn@math.stanford.edu \\ Sorin Popa \\ Department of Mathematics \\ University of California \\ Los Angeles, CA 90095-1555 \\ popa@math.ucla.edu
}

\author{
Vyjayanthi Chari \\ Department of Mathematics \\ University of California \\ Riverside, CA 92521-0135 \\ chari@math.ucr.edu \\ Kefeng Liu \\ Department of Mathematics \\ University of California \\ Los Angeles, CA 90095-1555 \\ liu@math.ucla.edu \\ Jie Qing \\ Department of Mathematics \\ University of California \\ Santa Cruz, CA 95064 \\ qing@ cats.ucsc.edu
}

\section{PRODUCTION}

Silvio Levy, Scientific Editor, production@msp.org

\section{SUPPORTING INSTITUTIONS}

ACADEMIA SINICA, TAIPEI

CALIFORNIA INST. OF TECHNOLOGY

INST. DE MATEMÁTICA PURA E APLICADA

KEIO UNIVERSITY

MATH. SCIENCES RESEARCH INSTITUTE

NEW MEXICO STATE UNIV.

OREGON STATE UNIV.

\author{
STANFORD UNIVERSITY \\ UNIV. OF BRITISH COLUMBIA \\ UNIV. OF CALIFORNIA, BERKELEY \\ UNIV. OF CALIFORNIA, DAVIS \\ UNIV. OF CALIFORNIA, LOS ANGELES \\ UNIV. OF CALIFORNIA, RIVERSIDE \\ UNIV. OF CALIFORNIA, SAN DIEGO \\ UNIV. OF CALIF., SANTA BARBARA
}

\author{
Daryl Cooper \\ Department of Mathematics \\ University of California \\ Santa Barbara, CA 93106-3080 \\ cooper@math.ucsb.edu \\ Jiang-Hua Lu \\ Department of Mathematics \\ The University of Hong Kong \\ Pokfulam Rd., Hong Kong \\ jhlu@maths.hku.hk \\ Paul Yang \\ Department of Mathematics \\ Princeton University \\ Princeton NJ 08544-1000 \\ yang@math.princeton.edu
}

These supporting institutions contribute to the cost of publication of this Journal, but they are not owners or publishers and have no responsibility for its contents or policies.

See inside back cover or msp.org/pjm for submission instructions.

The subscription price for 2015 is US \$420/year for the electronic version, and \$570/year for print and electronic.

Subscriptions, requests for back issues and changes of subscribers address should be sent to Pacific Journal of Mathematics, P.O. Box 4163, Berkeley, CA 94704-0163, U.S.A. The Pacific Journal of Mathematics is indexed by Mathematical Reviews, Zentralblatt MATH, PASCAL CNRS Index, Referativnyi Zhurnal, Current Mathematical Publications and Web of Knowledge (Science Citation Index).

The Pacific Journal of Mathematics (ISSN 0030-8730) at the University of California, c/o Department of Mathematics, 798 Evans Hall \#3840, Berkeley, CA 94720-3840, is published twelve times a year. Periodical rate postage paid at Berkeley, CA 94704, and additional mailing offices. POSTMASTER: send address changes to Pacific Journal of Mathematics, P.O. Box 4163, Berkeley, CA 94704-0163.

PJM peer review and production are managed by EditFLOW ${ }^{\circledR}$ from Mathematical Sciences Publishers.

\section{PUBLISHED BY}

\section{mathematical sciences publishers \\ nonprofit scientific publishing}

http://msp.org/

(C) 2015 Mathematical Sciences Publishers 


\title{
PACIFIC JOURNAL OF MATHEMATICS
}

\author{
Volume 279 No. 1-2 December 2015
}

In memoriam: Robert Steinberg

Robert Steinberg (1922-2014): In memoriam V. S. VARADARAJAN

Cellularity of certain quantum endomorphism algebras

HENNING H. ANDERSEN, GUSTAV I. LEHRER and RUIBIN ZHANG

Lower bounds for essential dimensions in characteristic 2 via orthogonal representations ANTONIO BABIC and VLADIMIR CHERNOUSOV

Cocharacter-closure and spherical buildings

Michael Bate, Sebastian Herpel, Benjamin Martin and Gerhard RöHrLe

Embedding functor for classical groups and Brauer-Manin obstruction

Eva Bayer-Fluckiger, Ting-Yu LeE and Raman Parimala

On maximal tori of algebraic groups of type $G_{2}$

Constantin Beli, PhilipPe Gille and Ting-Yu LeE

On extensions of algebraic groups with finite quotient

MICHEL BRION

Essential dimension and error-correcting codes

SHANE CERNELE and ZiNOVy REICHSTEIN

Notes on the structure constants of Hecke algebras of induced representations of finite Chevalley groups

Charles W. CuRTis

Complements on disconnected reductive groups

FRANÇOIS DIGNE and JEAN MICHEL

Extending Hecke endomorphism algebras

Jie Du, Brian J. Parshall and LeOnard L. SCOTT

Products of partial normal subgroups

ELLEN HENKE

Lusztig induction and $\ell$-blocks of finite reductive groups

RADHA KESSAR and GUNTER MALLE

Free resolutions of some Schubert singularities

Manoj Kummini, Venkatramani Lakshmibai, Pramathanath Sastry and C. S. Seshadri

Free resolutions of some Schubert singularities in the Lagrangian Grassmannian

VenKatramani LAKSHMibai and ReUVEN HODGES

Distinguished unipotent elements and multiplicity-free subgroups of simple algebraic groups

Martin W. Liebeck, Gary M. Seitz and Donna M. Testerman

Action of longest element on a Hecke algebra cell module

GEORGE LUSZTIG

Generic stabilisers for actions of reductive groups

BENJAMIN MARTIN

On the equations defining affine algebraic groups

VLADIMIR L. POPOV

Smooth representations and Hecke modules in characteristic $p$

PETER SCHNEIDER

On CRDAHA and finite general linear and unitary groups

BHAMA SRINIVASAN

Weil representations of finite general linear groups and finite special linear groups PHAM HUU TIEP

The pro- $p$ Iwahori Hecke algebra of a reductive $p$-adic group, $\mathrm{V}$ (parabolic induction) MARIE-FRANCE VIGNÉRAS

Acknowledgement 